Portland State University

PDXScholar

$1-1-2011$

\title{
Photoelectron Emission Control with Polarized Light in Plasmonic Metal Random Structures
}

Robert Campbell Word

Portland State University

Joseph Fitzgerald

Portland State University

Rolf Könenkamp

Portland State University, rkoe@pdx.edu

Follow this and additional works at: https://pdxscholar.library.pdx.edu/phy_fac

Part of the Physics Commons

Let us know how access to this document benefits you.

Citation Details

Word, R. C., Fitzgerald, J., \& Konenkamp, R. (2011). Photoelectron emission control with polarized light in plasmonic metal random structures. [Article]. Applied Physics Letters, 99(4), 3.

This Article is brought to you for free and open access. It has been accepted for inclusion in Physics Faculty Publications and Presentations by an authorized administrator of PDXScholar. Please contact us if we can make this document more accessible: pdxscholar@pdx.edu. 


\title{
Photoelectron emission control with polarized light in plasmonic metal random structures
}

\author{
R. C. Word, J. Fitzgerald, and R. Könenkamp ${ }^{\text {a) }}$ \\ Physics Department, Portland State University, Portland, Oregon 97201, USA
}

(Received 6 May 2011; accepted 28 June 2011; published online 26 July 2011)

\begin{abstract}
We report on the possibility of switching the emission rate of photoelectrons by polarization changes in the plasmon excitation light. Photoelectron emission is strongly enhanced in the nearfield of localized surface plasmons and occurs from areas with typical diameters of 20-70 nm. The underlying physical process involves excitation of a localized surface plasmon polariton with a femtosecond laser pulse, and a subsequent multi-photon photoemission process. The non-linearity of this process leads to a sharp polarization dependence that allows efficient switching of the emission. We demonstrate that a $90^{\circ}$ polarization change can result in on/off ratios of $\sim 100$ for electron emission. (C) 2011 American Institute of Physics. [doi:10.1063/1.3615783]
\end{abstract}

Surface plasmon polaritons have attracted wide interest for applications in ultrafast electronic and photonic devices, information devices, and for enhanced electron and light microscopy. The most efficient control approach in many of these applications is by a coherent interaction between excitation and plasmonic response. Coherent control has already been established in atomic and molecular physics and in semiconductor devices. ${ }^{1,2}$ It is now actively developed also in the area of plasmonics. ${ }^{3-5}$ Much research has focused on the intensity and pulse delay in lasers for control, but polarization control has also found attention, ${ }^{6}$ particularly in tip geometries for single molecule spectroscopy. ${ }^{7,8}$ When combined with plasmon-induced enhancement effects, coherent control is expected to allow the addressing of individual nanoscale systems and single molecules. Theoretical aspect of coherent control in nanoplasmonics has been discussed in several review articles. ${ }^{9,10}$

Here, we explore the possibility of using polarization properties of light to alter the spatial distribution of plasmonenhanced photoelectron emission in nanoscale metal random structures.

Metal random nanostructures were fabricated from $60 \mathrm{~nm}$ thick gold layers on conductive glass substrates by using ion milling with a focused $\mathrm{Ga}^{+}$beam in a FEI DB237 dual-beam system. The Au films were prepared by vacuum evaporation on 60-80 nm thick conductive indium-tin-oxide films on glass of $0.2 \mathrm{~mm}$ thickness. $\mathrm{Ga}^{+}$beam currents of $100 \mathrm{pA}$ and beam voltages of $30 \mathrm{kV}$ were used. The $\sim 3$ min ion milling reduced the average Au thickness unevenly and resulted in random structures as shown in Figure 1. Typically, the films have a gold coverage of $50 \%$, a fractal dimension of 1.30 , and a smallest feature size of $\sim 100 \mathrm{~nm} .{ }^{11} 790 \mathrm{~nm}$ light in 100-fs pulses at a frequency of $80 \mathrm{MHz}$ was used to excite localized surface plasmons in this structure, and an aberration-corrected photoemission electron microscope (PEEM) was used to visualize the spatial plasmon intensity distribution. ${ }^{12}$ The laser beam was polarized to $>99 \%$ linearity by a tunable wave plate and focused to an incident spot of about $100-\mu \mathrm{m}$

\footnotetext{
${ }^{\text {a) }}$ Author to whom correspondence should be addressed. Electronic mail: rkoe@pdx.edu.
}

diameter. The angle of incidence was $60^{\circ}$ to the electron microscope optical axis and surface normal.

The $790 \mathrm{~nm}$ light pulses illuminated the Au layer from the top side and indirectly from the bottom side (Fig. 1). In an area of $10 \times 10 \mu \mathrm{m}^{2}$, we can typically identify $\sim 1000$ localized photoemission areas. The pulsed light excites surface plasmons in the metal film. Due to the random morphology of the film, the plasmon response is localized, and very large non-propagating plasmon amplitudes are produced in some areas of the film. In these plasmon hot spots and in their nearfield region, a strong enhancement of photoelectron emission

(a)
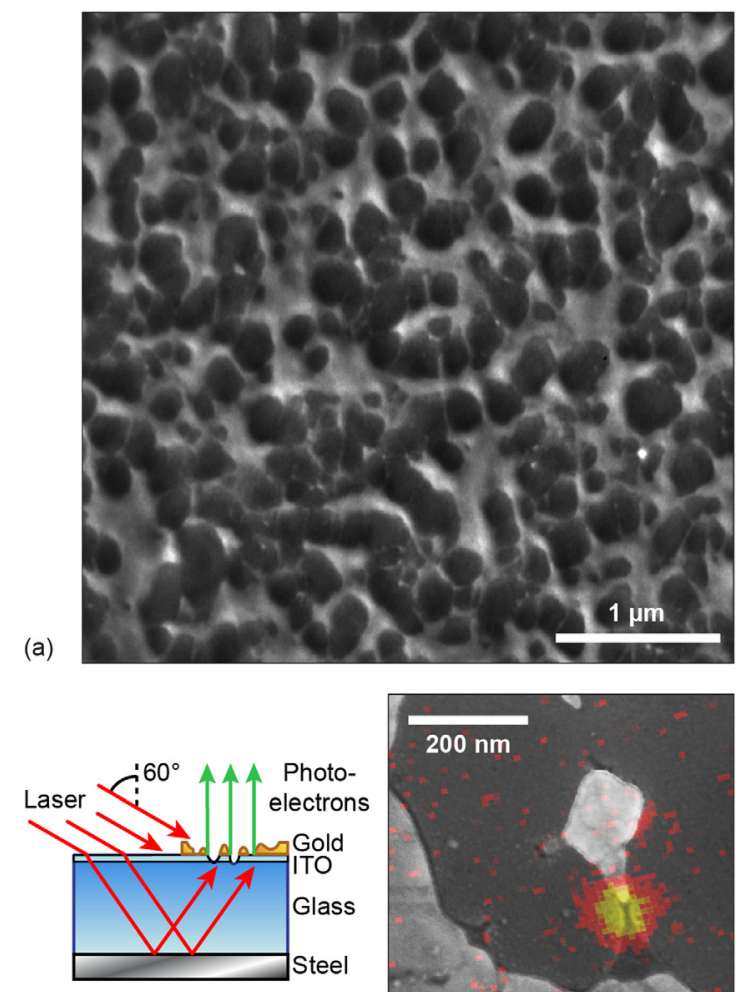

(b)

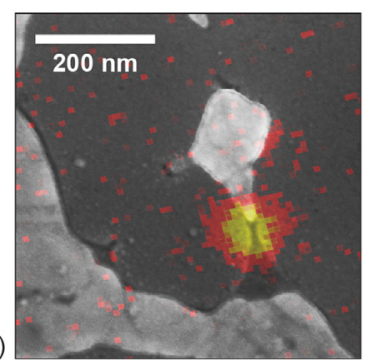

FIG. 1. (Color online) (a) Au fractal random structure-bright areas are gold and dark areas are ITO/glass; (b) illumination scheme for plasmon excitation and photoemission; and (c) photemission hot spot from indiumtin-oxide (ITO) in the gap of a plasmonic Au nanostructure. 
is observed. ${ }^{13-15}$ In our case, the photoemission occurs in a 3photon process that provides a quantum energy of $4.7 \mathrm{eV}$. This energy is sufficient for electron emission from the indium-tin-oxide (ITO), but insufficient for photoemission from the gold, which has a higher workfunction. As a consequence, the PEEM images predominantly show photoemission from ITO at the gold film edges as shown in Fig. 1(c).

Figure 2 shows examples of the photoemission hot spot spatial distribution and its polarization dependence. When the polarization angle of the excitation light is changed, strong changes in the hot spot location and intensities are observed. Part (b) of the figure shows the polarization dependence of two hot spot pairs, whose response is phase shifted by $90^{\circ}$. As a consequence of this phase shift, the two pairs are alternatively seen to be on or off. A simple basic understanding of the polarization dependence can be obtained by assuming that the gold nanostructure consists of antenna-like domains in which dipole characteristics dominate the multi-pole moments. Studies relating to the surface enhanced Raman effect have shown that the largest enhancement factors typically occur in the gaps of coupled metal junction. Figure 1(c) shows an example of this type of struc-

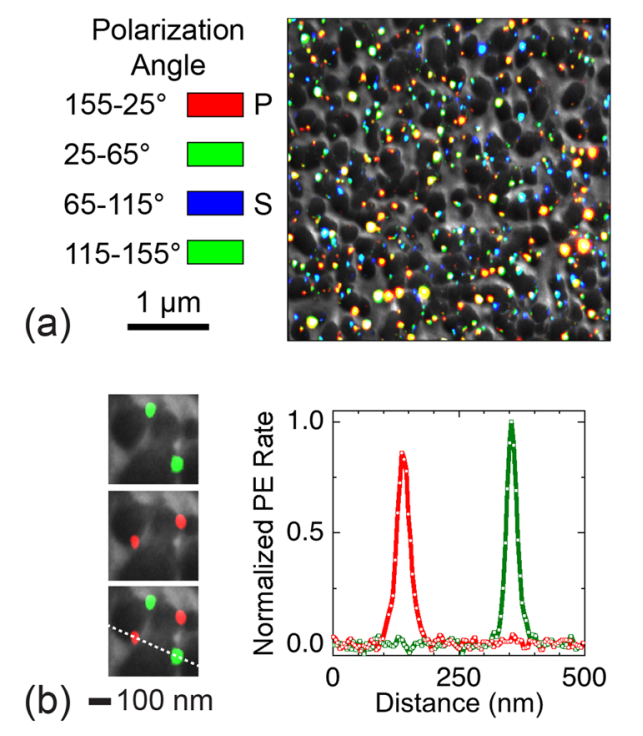

(c)

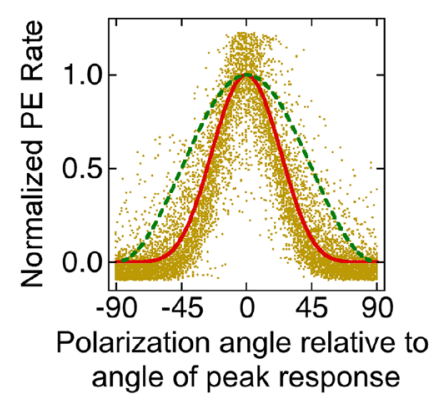

FIG. 2. (Color) (a) Photoemission hot spots in a $4 \times 4 \mu \mathrm{m}^{2}$ viewing area. The color of the hot spots relates to the polarization angle of the excitation light as specified in the legend to the left of the micrograph. (b) Two pairs of neighboring hot spots with differing polarization response. The red-marked hot spots emit at a polarization angle of $-45^{\circ}$, while the spots in green emit at $+45^{\circ}$. The intensity change upon $90^{\circ}$ polarization change at one location is of the order of 100. (c) Plot of the photoemission rate vs. polarization angle for 700 hot spots and a fitting of the experimental results with a $\cos ^{6} \theta$ function. $\mathrm{A} \cos ^{2} \theta$ function (broken line) is also provided for comparison. ture. In gap structures, the emission intensity is largest when the polarization is aligned across the gap. ${ }^{16-18}$ When the polarization and the cross-gap direction enclose an angle $\theta$, the electric field component across the gap is proportional to $\cos \theta$, and the intensity to $\cos ^{2} \theta$. The non-linear character of the 3-photon process brings in a third power dependence for the intensity, giving a $\cos ^{6} \theta$ dependence for the photoemission rate in this simple model. As is shown in Fig. 2(c), this power law gives a reasonable fit to the polarization dependence of the hot spots. From evaluating a large number of single hot spots, we find that the polarization dependence has a full width at half maximum of just $52^{\circ} \pm 4^{\circ}$, which is much smaller than that of a pure dipole response. This finding is somewhat surprising for a random structure, but may mainly be attributed to the large enhancement strength of dipolar gap structures. 3,19

The high power of the $\cos \theta$ dependence increases the switching contrast: A $90^{\circ}$ change in polarization angle comes along with a very strong emission intensity change. Evaluating Figure 2(b), we find that the polarization-induced on/off ratio is of the order of 100 at a given location. Thus, all photoemission hot spots can be switched effectively by polarization changes and the distribution seen in Figure 2(a) can completely be changed with a $90^{\circ}$ polarization change, i.e., hot spots which were "on" before the switching, are effectively "off" after switching, and a different non-overlapping hot spot pattern appears after a $90^{\circ}$ polarization change.

Polarization switching of infrared and visible light can be performed near the terahertz frequency range. ${ }^{20}$ In principle, there thus exists a highly effective control mechanism for plasmon-assisted photoelectron emission. Using different material combinations and optimizing surface, morphology and workfunction, may considerably increase the emission efficiency of these nanoscale, ultrafast electron sources.

Figure 3 shows a histogram for the number of hot spots for a given polarization of the excitation light based again on approximately 700 hot spots. We note a surprisingly strong deficiency in the number of hot spots that have their maximum emission rate for $\mathrm{p}$-polarized incident light. Most hot spots are

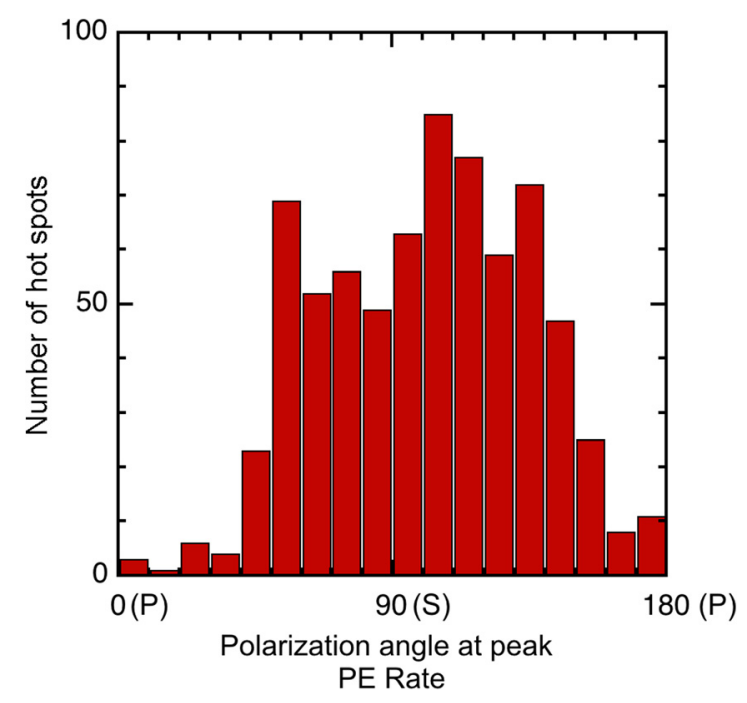

FIG. 3. (Color) Histogram of a population of 700 hot spots with their peak photoemission rates at various polarization angles of excitation light. 
instead responding to s-polarized light with electric field vector in the sample plane. We interpret this experimental result with two different excitation processes-one for p-polarized light that predominantly generates propagating surface plasmon modes in the Au sheet and, secondly, the excitation of localized modes that is not sensitive to the polarization angle. The results of Figure 3 thus indicate that the p-polarized light is mainly consumed by non-localized modes, which do not generate hot spots, ${ }^{21}$ while s-polarized light, which does not generate propagating modes due to momentum conservation rules, is responsible for most of the observed emission hot spots. ${ }^{22}$ The excitation of localized plasmon modes with spolarized light is therefore the main mechanism visible in these experiments.

To conclude, we found that the spatial distribution of photoelectron emission in random metal structures can effectively be controlled by the polarization of the excitation light. This somewhat surprising result is explained in terms of domains whose response has predominantly dipolar character defined by gap-like geometries. Due to the non-linearity in the underlying multi-photon process, the polarization dependence is even sharper than for an ideal dipole. We typically find a $\cos ^{6} \theta$ law to describe the polarization dependence. As a consequence, polarization changes of $90^{\circ}$ produce very large on/ off ratios at a photoemission hot spot. With the small scale of the spot, typically $20-70 \mathrm{~nm}$, and the fast response times of localized surface plasmons, typically $\sim 10 \mathrm{fs,} \mathrm{interesting} \mathrm{appli-}$ cations for fast nanoscale electron sources appear feasible.

The authors gratefully acknowledge assistance with the focused ion beam by T. Dornan at PSU. This research was supported by the Basic Science Office of the Department of Energy under Grant No. DEFG02-07ER46406.

${ }^{1}$ M. Shapiro and P. Brumer, Principles of the Quantum Control of Molecular Processes (Wiley-Interscience, Hoboken, NJ, 2003).

${ }^{2}$ S. A. Rice and M. Zhao, Optical Control of Molecular Dynamics (John Wiley \& Sons, New York, 2000).

${ }^{3}$ M. I. Stockman, S. V. Faleev, and D. J. Bergman, Phys. Rev. Lett. 88, 067402 (2002).

${ }^{4}$ A. Kubo, K. Onda, H. Petek, Z. Sun, Y. S. Jung, and H. K. Kim, Nano Lett. 5, 1123 (2005).

${ }^{5}$ Z.-W. Liu, Q.-H. Wei, and X. Zhang, Nano Lett. 5, 957 (2005).

${ }^{6}$ T. Brixner and G. Gerber, Opt. Lett. 26, 557 (2001).

${ }^{7}$ E. J. Sanchez, L. Novotny, and X. S. Xie, Phys. Rev. Lett. 82, 4014 (1999).

${ }^{8}$ T. Brixner, F. J. Garcia de Abajo, J. Schneider, and W. Pfeiffer, Phys. Rev. Lett. 95, 093901 (2005).

${ }^{9}$ S. Link and M. A. El-Sayed, Annu. Rev. Phys. Chem. 54, 331 (2003).

${ }^{10}$ E. Hutter and J. H. Fendler, Adv. Mater. 16, 1685 (2004).

${ }^{11}$ R. C. Word, T. Dornan, and R. Könenkamp, Appl. Phys. Lett. 96, 251110 (2010).

${ }^{12}$ R. Könenkamp, R. C. Word, G. F. Rempfer, T. Dixon, L. Almaraz, and T. Jones, Ultramicroscopy 110, 899 (2010).

${ }^{13}$ J. Kupersztych and M. Raynaud, Phys. Rev. Lett. 95, 147401 (2005).

${ }^{14}$ T. Tsang, T. Srinivasan-Rao, and J. Fischer, Opt. Lett. 15, 866 (1990).

${ }^{15}$ H. Chen, J. Boneberg, and P. Leiderer, Phys. Rev. B 47, 9956 (1993).

${ }^{16}$ H. X. Xu and M. Kall, ChemPhysChem 4, 1001 (2003).

${ }^{17}$ P. G. Etchegoin, C. Galloway, and E. C. Le Ru, Phys. Chem. Chem. Phys. 8, 2624 (2006).

${ }^{18}$ H. Wei and F. Hao, Nano Lett. 8, 2497 (2008).

${ }^{19}$ B. Hecht, H. Bielefeldt, L. Novotny, Y. Inouye, and D. W. Pohl, Phys. Rev. Lett. 77, 1889 (1996).

${ }^{20}$ W. J. Johnston, J. P. Prineas, and A. L. Smirl, J. Appl. Phys. 101, 046101 (2007).

${ }^{21}$ L. Cao, R. A. Nome, J. M. Montgomery, S. K. Gray, and N. F. Scherer, Nano Lett. 10, 3389 (2010).

${ }^{22}$ X.-R. Su, Z.-S. Zhang, L.-H. Zhang, Q.-Q. Li, C.-C. Chen, Z.-J. Yang, and Q.-Q. Wang, Appl. Phys. Lett. 96, 043113 (2010). 